PROCEEDINGS OF THE

AMERICAN MATHEMATICAL SOCIETY

Volume 130, Number 8, Pages 2479-2485

S 0002-9939(02)06323-2

Article electronically published on February 12, 2002

\title{
THE KAUFFMAN BRACKET SKEIN AS AN ALGEBRA OF OBSERVABLES
}

\author{
DOUG BULLOCK, CHARLES FROHMAN, AND JOANNA KANIA-BARTOSZYŃSKA
}

(Communicated by Ronald A. Fintushel)

\begin{abstract}
We prove that the Kauffman bracket skein algebra of a cylinder over a surface with boundary, defined over complex numbers, is isomorphic to the observables of an appropriate lattice gauge field theory.
\end{abstract}

\section{INTRODUCTION}

Lattice gauge field theory brings the representation theory of an underlying manifold and its quantum invariants into the same setting. Consider the case of a cylinder over a compact, oriented surface with boundary. A lattice model of the surface determines an algebra of gauge invariant fields (i.e. observables). In the classical case, based on a connected, simply connected Lie group $G$, observables are the characters of the fundamental group of the lattice represented in $G$. Wilson loops can be understood as traces of conjugacy classes in the fundamental group of the lattice. For the theory based on a Drinfeld-Jimbo deformation of a simple Lie algebra $\mathfrak{g}$, the observables are a deformation quantization of the $G$-characters of the surface with respect to the standard Poisson structure 2]. In the case of $U_{h}\left(s l_{2}\right)$ this, together with the classical isomorphism [1] 6], allowed us to prove that the algebra of observables is the Kauffman bracket skein algebra of the surface, completed as an algebra over formal power series.

In this paper we return to an analytic setting in which the deformation parameter is any complex number other than a root of unity. The analogous theorem relating observables and the Kauffman bracket skein algebra is again true. The proof is based on the combinatorial equivalence between the Temperley-Lieb algebra and the quantized invariant theory of $S L_{2}$; it does not explicitly use the relationship with surface characters.

The paper is organized as follows. Section 2 recalls definitions and associated formulas of the Kauffman bracket skein algebra. Section 3 summarizes, for the generic parameter, the construction of a quasi-triangular matrix model of quantum $S L_{2}$. Section 4 outlines basic definitions and constructions of lattice gauge field theory. Section 5 describes the correspondence between skeins and intertwiners in the Verlinde algebra for quantum $S L_{2}$. Finally, Section 6 contains a proof of the main theorem.

Received by the editors November 6, 2000 and, in revised form, March 16, 2001.

2000 Mathematics Subject Classification. Primary 57M27, 81T13.

This research was partially supported by an NSF-DMS Postdoctoral Research Fellowship, and by NSF grants DMS-9803233 and DMS-9971905. 


\section{KAUfFMAN BRACKET SKEIN ALGEBRA}

Let $t$ be a complex number that is neither 0 nor a root of unity. Suppose that $F$ is a compact, oriented surface with boundary and $I$ is a closed interval. Denote by $\mathcal{L}$ the set of isotopy classes of framed links in $F \times I$, including the empty link. Let $\mathbb{C} \mathcal{L}$ be the vector space with basis $\mathcal{L}$. Define $S_{t}$ to be the subspace of $\mathbb{C} \mathcal{L}$ spanned by all expressions of the form $\left.\backslash-t \cup-t^{-1}\right)$ ( and $\bigcirc+t^{2}+t^{-2}$, where the framed links in each expression are identical outside the balls pictured in the diagrams.

The Kauffman bracket skein algebra $K_{t}(F \times I)$ is the quotient $K_{t}(F \times I)=$ $\mathbb{C} \mathcal{L} / S_{t}$. Multiplication is given by laying one link over the other. More precisely, if $\alpha$ and $\beta$ are in $\mathcal{L}$, isotop them so that $\alpha$ lies in $F \times\left[0, \frac{1}{2}\right)$, and $\beta$ in $F \times\left(\frac{1}{2}, 1\right]$. Then $\alpha * \beta$ is the union of these two links in $F \times[0,1]$. Extend linearly to a product on $\mathbb{C} \mathcal{L}$. Since $S_{t}$ is an ideal, the product descends, making the skein module into a skein algebra. Since the algebra structure depends on the specific product structure of $F \times I$, rather than its topological type, we use the notation $K_{t}(F)$.

We use the standard convention of modeling a skein in $K_{t}(F)$ on a framed, admissibly colored, trivalent graph. An admissible coloring is an assignment of a nonnegative integer to each edge so that the colors at each vertex form admissible triples. A triple $(a, b, c)$ is admissible if $a \leq b+c, b \leq a+c, c \leq a+b$ and $a+b+c$ is even. The corresponding skein in $K_{t}(F)$ is obtained by replacing each edge labeled with the letter $m$ by the $m$-th Jones-Wenzl idempotent (see [7, or [5, p. 136]), and replacing trivalent vertices with Kauffman triads (see [5, Fig. 14.7]). If $s$ is a trivalent spine of $F$, then the set of skeins carried by admissible colorings of $s$ forms a basis $\mathcal{B}_{1}$ for $K_{t}(F)$. If $F$ is an annulus, $\mathcal{B}_{1}$ consists of skeins obtained by labeling the core with a Jones-Wenzl idempotent. One may think of the core as a "trivalent" spine with one vertex, whose admissible labels are $\{(n, n, 0)\}$. The space $K_{t}(F)$ also has a basis $\mathcal{B}_{2}$ consisting of all links with simple diagrams on $F$, i.e. with no crossings and no trivial components.

\section{Representations}

The details of the following can be found in $\left[3\right.$. Let $\mathcal{A}_{t}$ be the unital Hopf algebra on $X, Y, K, K^{-1}$, with relations:

$$
\begin{gathered}
K X=t^{2} X K, \quad K Y=t^{-2} Y K, \\
X Y-Y X=\frac{K^{2}-K^{-2}}{t^{2}-t^{-2}}, \quad K K^{-1}=1 .
\end{gathered}
$$

Let $\underline{m}$ denote the irreducible $(m+1)$-dimensional representation of $\mathcal{A}_{t}$. Fixing an ordered basis for $\underline{m}$ we define linear functionals ${ }^{m} c_{j}^{i}: A_{t} \rightarrow \mathbb{C}$ to be the coefficient in the $i$-th row and $j$-th column in the representation $\underline{m}$. The ${ }^{m} c_{j}^{i}$ form a basis for the stable subalgebra ${ }_{q} S L_{2}$ of the Hopf algebra dual $A_{t}^{o}$. (Here $q=t^{4}$.) Define

$$
\overline{\mathcal{A}}_{t}=\prod_{m=0}^{\infty} M_{m+1}(\mathbb{C})
$$

and give it the product topology. A typical element of $\overline{\mathcal{A}}_{t}$ is a sequence of arbitrarily chosen matrices in which the $i$-th term is an $(i+1) \times(i+1)$ matrix.

Let $\rho_{m}: \mathcal{A}_{t} \rightarrow M_{m+1}(\mathbb{C})$ be the homomorphism corresponding to the representation $\underline{m}$. The homomorphism

$$
\Theta: \mathcal{A}_{t} \rightarrow \overline{\mathcal{A}}_{t}
$$


given by $\Theta(Z)=\left(\rho_{0}(Z), \rho_{1}(Z), \rho_{2}(Z), \ldots\right)$, is injective and its image is dense in $\overline{\mathcal{A}}_{t}$ (see [3]). The algebra $\overline{\mathcal{A}}_{t}$ is the completion of $\mathcal{A}_{t}$ by equivalence classes of Cauchy sequences in the weak topology from ${ }_{q} S L_{2}$. It has the structure of a topological ribbon Hopf algebra. The projection of $\overline{\mathcal{A}}_{t}$ onto its $(m+1)$-st factor is an irreducible representation of $\overline{\mathcal{A}}_{t}$, also denoted by $\underline{m}$. Composing ${ }^{m} c_{j}^{i}$ with this projection yields a function on $\overline{\mathcal{A}}_{t}$, also called ${ }^{m} c_{j}^{i}$. Thus ${ }_{q} S L_{2}$ is understood to lie in $\left(\overline{\mathcal{A}}_{t}\right)^{o}$.

\section{Lattice gauge Field theory}

In this section we recall basic definitions and constructions of lattice gauge field theory. Details can be found in [2].

Let $\Gamma$ be an oriented, ciliated graph; i.e. the edges are oriented and the vertices carry a linear ordering of the adjacent edges. One can think of this as instructions for building a strip and disk model of an oriented surface $F$ having $\Gamma$ as a strong deformation retract. Vertices correspond to the disks, edges to strips, and the ciliation determines how to glue the strips to the disks. The surface $F$ is called the envelope of $\Gamma$. Define a space of connections

$$
\mathbb{A}(\Gamma)=\bigotimes_{\text {edges } e}\left(\overline{\mathcal{A}}_{t}\right)_{e}
$$

and define an algebra of fields

$$
C[\mathbb{A}(\Gamma)]=\bigotimes_{\text {edges } e}\left({ }_{q} S L_{2}\right)_{e} .
$$

Note that fields are functions on connections in the obvious way. The connections form a coalgebra with comultiplication as defined in 2. Multiplication of fields is the convolution product dual to comultiplication of connections. There is an action of the gauge algebra,

$$
\mathcal{G}(\Gamma)=\bigotimes_{\text {vertices } v}\left(\overline{\mathcal{A}}_{t}\right)_{v}
$$

on the space of connections, and adjointly on fields. The invariant part of the gauge fields under this action is called the observables, $\mathcal{O}(\Gamma)$. The multiplication of fields restricts to make $\mathcal{O}(\Gamma)$ into an algebra, which is a deformation of the $S L_{2}(\mathbb{C})$-characters of $\pi_{1}(F)$.

Let $V$ be a representation of the Hopf algebra $\overline{\mathcal{A}}_{t}$, that is, $V$ is a finite dimensional left module over $\overline{\mathcal{A}}_{t}$.

The dual vector space to $V$ carries two distinct $\overline{\mathcal{A}}_{t}$-module structures. When $\overline{\mathcal{A}}_{t}$ acts on the left, the dual module is denoted by $V^{*}$. The action is

$$
Z \cdot \phi(v)=\phi(S(Z) \cdot v)
$$

for any $Z \in \overline{\mathcal{A}}_{t}, v \in V$ and $\phi$ in the dual vector space to $V$. When $\overline{\mathcal{A}}_{t}$ acts on the right, denote the dual by $V^{\prime}$ with

$$
\phi(v) \cdot Z=\phi(Z \cdot v)
$$

There is an alternate description of observables in terms of "colorings" of the lattice. Assigning a representation $V_{e}$ to each positively oriented edge $e$ of the lattice $\Gamma$ determines a map

$$
\mathbb{A}(\Gamma) \rightarrow \bigotimes_{e}\left(V_{e}^{*} \otimes V_{e}\right)
$$


This yields, at each vertex, a tensor product of representations coming from the edges adjoining that vertex taken in the order given by the ciliation. Use the representation $V_{e}$ for the edges $e$ starting at a vertex and the dual $V_{e}^{*}$ for the edges terminating there. The resulting representation at a vertex $v$ is denoted by $V_{v}$. Finally, choose $\phi_{v} \in \operatorname{Inv}\left(V_{v}^{\prime}\right)$ for each vertex $v$. The element $\bigotimes_{v}\left(\phi_{v}\right)$ is evaluated on a connection $\bigotimes_{e} x_{e}$ by mapping $\bigotimes_{e} x_{e}$ to $\bigotimes_{e}\left(V_{e}^{*} \otimes V_{e}\right)$ and then re-parsing to an element of $\bigotimes_{v} V_{v}$. By [2, Corollary 1] every observable is a linear combination of observables of this form.

Now assume that $\Gamma$ is a trivalent lattice colored by irreducible representations. The coloring is admissible if, at each vertex, the integers corresponding to the colorings of the incident edges form an admissible triple.

Proposition 1. Suppose that $\Gamma$ is an admissibly colored trivalent lattice. For each $V_{v}$ there exists a non-zero dual element invariant under the right action of $\overline{\mathcal{A}}_{t}$. The tensor product of these invariants over all vertices defines an observable.

The set of such observables, one for each admissible coloring, is a basis for $\mathcal{O}(\Gamma)$.

Proof. Let $c=\left\{\underline{m}_{e} \mid e\right.$ is an edge of $\left.\Gamma\right\}$ be an admissible coloring. Note that admissibility implies a 1-dimensional invariant subspace in each $V_{v}^{\prime}$. Hence there is a non-zero observable $o_{c}=\bigotimes_{v} \phi_{v}$. Since $o_{c}$ is nonzero, there exists $X_{c} \in \bigotimes_{v} V_{v}$ so that $o_{c}\left(X_{c}\right) \neq 0$.

Let $P: \bigotimes_{v} V_{v} \rightarrow \bigotimes_{e}\left(\underline{m}_{e}^{*} \otimes \underline{m}_{e}\right)$ be the "parsing" map. Let $\iota_{m}$ be the map

$$
\underline{m}^{*} \otimes \underline{m} \cong M_{m+1}(\mathbb{C}) \rightarrow \prod_{n} M_{n}(\mathbb{C})=\overline{\mathcal{A}}_{t},
$$

where the inclusion is given by forming a sequence that has all zero matrices except for the $(m+1)$-st entry corresponding to $\underline{m}$.

Define $x_{c}$ to be the connection $\left(\bigotimes_{e} \iota_{m_{e}}\right)\left(P\left(X_{c}\right)\right)$. Clearly $o_{c}\left(x_{c}\right) \neq 0$. Since $\rho_{m} \circ \iota_{n}=0$ unless $m=n, x_{c}$ is annihilated by all observables constructed from colorings different than $c$.

From this we conclude that any set of observables constructed from distinct colorings is independent. By [2] they span.

There is a map

$$
\Phi: K_{t}(F) \rightarrow \mathcal{O}(\Gamma)
$$

that assigns to each framed link a Wilson operator. Classically, a Wilson operator is the trace of the holonomy of a connection along some fixed loop. The construction in the quantum setting is described in [2]. In a theory based on the DrinfeldJimbo $U_{h}\left(s l_{2}\right)$ and its Hopf algebra dual, the observables are isomorphic to the Kauffman bracket skein algebra of the lattice envelope. Since $U_{h}\left(s l_{2}\right)$ is a complete topological algebra over $\mathbb{C}[[h]]$, it was necessary to complete the skein algebra as well. The isomorphism was inferred from the classical isomorphism, the agreement of Poisson brackets, and the $h$-adic completion. In Section 6 we will show directly that the map $\Phi$ is an isomorphism.

\section{Temperley-Lieb THEORY}

In this section we recall the correspondence between skeins and intertwiners in the category of representations $\underline{m}$ of $\overline{\mathcal{A}}_{t}$.

Consider a rectangle $R=I \times I$ with $2 n$ distinguished points: $n$ of them on $I \times\{0\}$ and $n$ on $I \times\{1\}$. Take the space of blackboard framed tangles with $n$ 
arc components ending at the distinguished points. Its quotient by the Kauffman bracket skein relations is denoted by $K_{t}(R, n)$. This quotient has an algebra structure given by placing the bottom of a rectangle on the top of another in such a way that the distinguished points meet.

Fact 1. The algebra $K_{t}(R, n)$ is isomorphic to $\operatorname{End} \overline{\mathcal{A}}_{t}\left(\underline{1}^{\otimes n}\right)$, the space of $\overline{\mathcal{A}}_{t}$-linear maps of $\underline{1}^{\otimes n}$ to itself.

In the basis $\left\{e_{1 / 2}, e_{-1 / 2}\right\}$ of $\underline{1}$, the isomorphism is given by a tangle functor which makes the following assignments. A local maximum is sent to the morphism $\mu: \underline{1} \otimes \underline{1} \rightarrow \underline{0}$ defined by

$$
\begin{gathered}
\mu\left(e_{1 / 2} \otimes e_{-1 / 2}\right)=i t, \quad \mu\left(e_{-1 / 2} \otimes e_{1 / 2}\right)=-i t^{-1}, \\
\mu\left(e_{-1 / 2} \otimes e_{-1 / 2}\right)=\mu\left(e_{1 / 2} \otimes e_{1 / 2}\right)=0 .
\end{gathered}
$$

A local minimum is associated to the morphism $\eta: \underline{0} \rightarrow \underline{1} \otimes \underline{1}$ given by

$$
\eta(1)=i t e_{1 / 2} \otimes e_{-1 / 2}-i t^{-1} e_{-1 / 2} \otimes e_{1 / 2} .
$$

Fact 2. The isomorphism takes the $n$-th Jones-Wenzl idempotent to the intertwiner that projects $\underline{1}^{\otimes n}$ onto its highest weight invariant subspace.

Let $\mathbb{H}=\{(x, y) \mid y \geq 0\}$ be the closed upper half plane. For any $n$, choose $2 n$ distinguished points on the $x$-axis, $\{(1,2, \ldots, 2 n)\}$, and form a space of blackboardframed tangles with $n$ arc components ending at the distinguished points. The quotient of this space by the Kauffman bracket skein relations is denoted $K_{t}(\mathbb{H}, 2 n)$.

Fact 3. $K_{t}(\mathbb{H}, 2 n) \cong \operatorname{Inv}\left(\left((\underline{1} \otimes \underline{1})^{\otimes n}\right)^{\prime}\right)$.

The isomorphism is given by the same tangle functor as for Fact 1

An admissible triple $(m, n, p)$ determines a skein in $K_{t}(\mathbb{H}, m+n+p)$ consisting of a Kauffman triad with all three legs attached to the $x$-axis. Fact 2 gives a canonical inclusion of $\underline{m} \otimes \underline{n} \otimes \underline{p}$ into $\underline{1}^{\otimes m} \otimes \underline{1}^{\otimes n} \otimes \underline{1}^{\otimes p} \cong(\underline{1} \otimes \underline{1})^{\otimes(m+n+p) / 2}$.

Fact 4. An admissible triple $(m, n, p)$ - equivalently, a Kauffman triad - corresponds to a nonzero vector in the 1-dimensional space $\operatorname{Inv}\left((\underline{m} \otimes \underline{n} \otimes \underline{p})^{\prime}\right)$.

\section{Observables and the Kauffman Bracket skein Algebra}

Our goal is to prove a theorem analogous to [2, Theorem 10], but replacing power series by complex numbers.

Theorem 1. Let $\Gamma$ be a lattice and let $F$ be its envelope. Assume that $t \in \mathbb{C} \backslash\{0\}$ is not a root of unity. The algebra of observables of lattice gauge field theory on $\Gamma$ based on $\left(\overline{\mathcal{A}}_{t},{ }_{q} S L_{2}\right)$ is isomorphic to $K_{t}(F)$.

Proof. From [2] we have an algebra map from $\mathbb{C} \mathcal{L}$ to $\mathcal{O}(\Gamma)$ taking a link to the corresponding Wilson operator. As in [2, Theorem 10] this map descends to $\Phi$ : $K_{t}(F) \rightarrow \mathcal{O}(\Gamma)$.

The following description of $\Phi$ is implicit in [2]. Since $\mathcal{O}(\Gamma)$ is a homeomorphism invariant of $F$, we can assume that the lattice $\Gamma$ comes from giving orientation and ciliation to a trivalent spine $\gamma$ of $F$. Let $L$ be an element of the basis $\mathcal{B}_{2}$ of $K_{t}(F)$ (i.e., a link with a simple diagram). Choose an orientation of $L$. To compute the image of $L$ under $\Phi$, first perform the composition

$$
\bigotimes_{e} \overline{\mathcal{A}}_{t} \stackrel{\Delta}{\longrightarrow} \bigotimes_{e} \overline{\mathcal{A}}_{t}^{\otimes n(e)} \stackrel{\otimes_{e} \rho_{1}^{\otimes n(e)}}{\longrightarrow} \bigotimes_{e}\left(\underline{1}^{*} \otimes \underline{1}\right)^{\otimes n(e)} .
$$




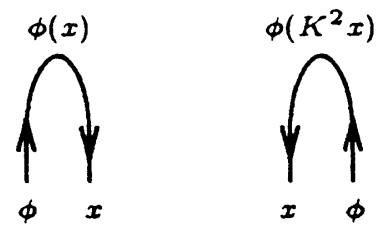

FiguRE 1. Cap tangles

Here $n(e)$ is the number of strands of $L$ running along the edge $e$ of $\Gamma$, and $\Delta$ means comultiplying $n(e)-1$ times in the factor corresponding to $e$.

Second, in each factor where the corresponding segment of $L$ runs against the orientation of the edge of $\Gamma$, apply $i D \otimes i D^{-1}: \underline{1}^{*} \otimes \underline{1} \rightarrow \underline{1} \otimes \underline{1}^{*}$. Here, the morphism $D: \underline{1}^{*} \rightarrow \underline{1}$ is defined by

$$
D\left(e^{1 / 2}\right)=i t e_{-1 / 2}, \quad D\left(e^{-1 / 2}\right)=-i t^{-1} e_{1 / 2} .
$$

Third, treat each ciliated vertex as a half plane with the cilium at infinity. Up to isotopy, the link $L$ now appears as a collection of oriented caps in each half plane. The cap pictured on the left of Figure 1 is associated with the map $\underline{1}^{*} \otimes \underline{1} \rightarrow \mathbb{C}$ where $\phi \otimes x \mapsto \phi(x)$, and $\underline{1} \otimes \underline{1}^{*} \rightarrow \mathbb{C}$ is given by $x \otimes \phi \mapsto \phi\left(K^{2} x\right)$ for the cap on the right.

Finally, to obtain $\Phi(L)$, multiply the result by $(-1)^{|L|}$, where $|L|$ denotes the number of components of $L$.

Notice that $\rho_{1}$ sends the switch map of [2] to the map $i D \otimes i D^{-1}$ and sends multiplications to contractions. Hence our description of $\Phi(L)$ for $L \in \mathcal{B}_{2}$ coincides with the Wilson operator. It follows from [2] that $\Phi$ does not depend on the choice of orientation of $L$. Extend it linearly to all of $K_{t}(F)$. It is a homomorphism of algebras $K_{t}(F)$ and $\mathcal{O}(\Gamma)$. In order to prove that $\Phi$ is an isomorphism we factor it into two maps which are isomorphisms on the level of vector spaces.

The first map, expressed in the basis $\mathcal{B}_{2}$, is given by a diagonal matrix with 1's and -1 's on the diagonal. The second map,

$$
\Phi_{u}: K_{t}(F) \rightarrow \mathcal{O}(\Gamma),
$$

does not require a choice of orientation of a link $L$. To compute the image of $L \in \mathcal{B}_{2}$ under $\Phi_{u}$ first perform the composition (4). Second, apply the map $D$ to each copy of $\underline{1}^{*}$. Third, treat each vertex as a half plane and associate the map $\mu$ from equation (3) to the (unoriented) caps.

Checking all possible orientations of $L$ and $\Gamma$ shows that $\Phi(L)= \pm \Phi_{u}(L)$.

By Fact 4 , the map $\Phi_{u}$ takes an element of the basis $\mathcal{B}_{1}$ (i.e., a skein obtained by an admissible coloring of $\gamma$ ) to an observable coming from coloring the edges of $\Gamma$ with corresponding irreducible representations of $\overline{\mathcal{A}}_{t}$. Thus, by Proposition 1 , the map $\Phi_{u}$ takes the basis $\mathcal{B}_{1}$ of $K_{t}(F)$ to a basis of $\mathcal{O}(\Gamma)$.

As $\Phi$ and $\Phi_{u}$ differ by a composition with an isomorphism, both maps are isomorphisms.

\section{REFERENCES}

[1] D. Bullock, Rings of $S L_{2}(\mathbb{C})$-characters and the Kauffman bracket skein module, Comment. Math. Helv. 72 (1997), 521-542. MR 98k:57008 
[2] D. Bullock, C. Frohman, J. Kania-Bartoszyńska, Topological interpretations of Lattice Gauge Field Theory, Comm. Math. Phys. 198 (1998) 47-81. MR 2000b:81107

[3] C. Frohman, J. Kania-Bartoszyńska, A matrix model for quantum $S L_{2}$, preprint, math.QA/0010328.

[4] L. H. Kauffman and S. Lins, Temperley-Lieb recoupling theory and invariants of 3-manifolds, Ann. of Math. Studies 143, Princeton University Press (1994). MR 95c:57027

[5] W. B. R. Lickorish, An Introduction to Knot Theory, Springer, GTM 175, 1997. MR 98f:57015

[6] J. H. Przytycki, A. S. Sikora, On skein algebras and $S L_{2}(\mathbb{C})$-character varieties, Topology 39(1) (2000), 115-148. MR 2000g:57026

[7] H. Wenzl, On sequences of projections, C.R. Math. Rep. Acad. Sci. Canada IX, (1987) 5-9. MR 88k:46070

Department of Mathematics, Boise State University, Boise, Idaho 83725

E-mail address: bullock@math.boisestate.edu

Department of Mathematics, University of Iowa, Iowa City, Iowa 52242

E-mail address: frohman@math.uiowa.edu

Department of Mathematics, Boise State University, Boise, Idaho 83725

E-mail address: kania@math.boisestate.edu 